\title{
Self-Reported Adherence to Oral Endocrine Therapy among Breast Cancer Patients in Jordan
}

\section{Dear Editor,}

Non-adherence to medications is a significant health issue, as it can reduce the efficacy of therapeutic management and increase the likelihood of relapse. ${ }^{1}$ Ensuring that patients with breast cancer $(\mathrm{BC})$ adhere to the prescribed duration of endocrine therapy (ET) is, however, difficult due to the long duration of treatment. Several studies have evaluated adherence to ET. ${ }^{2-4}$ To the best of the authors' knowledge, no studies have been conducted in the Middle East to evaluate adherence to ET. Subsequently, the authors conducted a study to evaluate the level of adherence to ET among patients with BC. The study also aimed to describe the patients' perceptions of ET and associations between adherence and patient-related factors.

This study was conducted between October 2017 and July 2018 at King Hussein Cancer Center, Amman, Jordan and involved the administration of a questionnaire to patients being treated for BC. Patients were recruited at the hospital's outpatient pharmacy and the chemotherapy infusion clinic. Those who had a refill for ET (tamoxifen, anastrazole, examastaine or letrazole) and had been on therapy for at least one month were included.

The questionnaire was developed by a survey specialist at the institution since, to the best of the authors' knowledge, there was no validated, well-established questionnaire in Arabic. The questionnaire contained items that were reported by Pourcelot et al. ${ }^{2}$ The questionnaire included questions on adherence (the number of missed doses of ET during the previous month) and type and duration of ET as well as perception about ET. The survey specialist and the clinical investigators assessed the items for clarity and relevance to the patients to establish the face and content validity of the questionnaire. The questionnaire was pilot-tested in a small group of patients for one week and modified based on the feedback received.

Continuous data were presented as means \pm standard deviation, while categorical data were presented as numbers and percentages. A $P \leq 0.05$ was considered statistically significant. Analysis of variance test was used to determine any associations between adherence and continuous data while chi-squared test was used for categorical data.

Ethical approval was granted for this study by the institutional review board (17 KHCC 93). The questionnaire contained a statement in the first page which was considered a consent form; all included patients provided informed consent.

A total of 202 questionnaires were completed. The mean age of patients was $45.8 \pm 10.7$ years [Table 1]. In the sample, there were 113 patients $(55.9 \%)$ on tamoxifen, 34 (16.8\%) on letrazole, $36(17.8 \%)$ on anastrazole and $10(5.0 \%)$ on exemastane. Four patients (2.0\%) did not know the name of the ET they were taking, and five patients (2.5\%) did not answer the question. ET had been taken for $\leq 6$ months by 59 patients $(29.2 \%)$, for $6-12$ months by 51 patients (25.2\%), for $1-3$ years by 77 patients (38.1\%) and for $3-5$ years by 15 patients $(7.4 \%)$.

In terms of patients' perceptions about ET, approximately two-thirds (62.9\%) reported that they didn't know to what extent they needed ET and approximately half of the patients (47.2\%) didn't know whether ET could help in reducing recurrence of $\mathrm{BC}$. However, the majority of the patients were aware of their treatment plan (89.9\%) and reported understanding of their disease (90.8\%). Most of the patients (86.1\%) reported that they had received information on the importance of ET from their physicians but only half of the patients (52.0\%) reported having received medication counselling from a pharmacist. The only factor that was significantly associated with adherence was the duration of treatment $(P=0.048)$; the shorter the duration of ET, the fewer reported missed doses.

This study found a relatively high rate of adherence to ET. Patients were considered adherent if they took at least $80 \%$ of their prescribed medication over a certain period. ${ }^{5}$ The majority of patients in the current study (99.5\%) reported adherence rates of $80 \%$ or more over one month. Studies have reported high as well as low rates of adherence. . $^{2,3,5}$

In the current study, most participants reported that they had received information on the importance of ET, but this information was mostly from their physicians rather than pharmacists. In addition, no association was found between the high adherence and the information provided by physicians; however, this may be due to the nature of the study as there was no objective measure of the physician's role in the patient's counselling. The 
Table 1: Characteristics of patients with breast cancer on endocrine therapy in Jordan $(\mathrm{N}=202)$

\begin{tabular}{|c|c|}
\hline Characteristic & n (\%) \\
\hline \multicolumn{2}{|l|}{ Marital status $(n=200)$} \\
\hline Married & $163(81.5)$ \\
\hline Single & $19(9.5)$ \\
\hline Widowed & $13(6.5)$ \\
\hline Divorced & $5(2.5)$ \\
\hline \multicolumn{2}{|l|}{ Educational level $(n=199)$} \\
\hline Bachelor's degree & $59(29.6)$ \\
\hline High school & $58(29.1)$ \\
\hline Diploma & $51(25.6)$ \\
\hline Master's or doctoral degree & $13(6.5)$ \\
\hline Others & $18(9.0)$ \\
\hline \multicolumn{2}{|l|}{ Area of residence $(n=190)$} \\
\hline Amman & $127(66.8)$ \\
\hline Outside Amman & $55(28.9)$ \\
\hline Outside Jordan & $8(4.2)$ \\
\hline \multicolumn{2}{|c|}{ Family income per month in Jordanian Dinar $(n=190)$} \\
\hline$<500$ & $98(51.6)$ \\
\hline $500-1000$ & $72(37.9)$ \\
\hline $1000-2000$ & $16(8.4)$ \\
\hline$>2000$ & $4(2.1)$ \\
\hline \multicolumn{2}{|l|}{ Nationality $(n=199)$} \\
\hline Jordanian & $179(89.9)$ \\
\hline Other & $20(10.1)$ \\
\hline \multicolumn{2}{|c|}{ Family history of breast cancer $(n=199)$} \\
\hline Yes & $55(27.6)$ \\
\hline No & $144(72.4)$ \\
\hline \multicolumn{2}{|c|}{ On other medications $(n=187)$} \\
\hline Yes & $55(29.4)$ \\
\hline No & $132(70.6)$ \\
\hline
\end{tabular}

only factor associated with adherence was the duration of treatment. A similar association was reported by Robinson et al., who found that the rate of adherence decreased from year one of treatment to year five by $50 \% .{ }^{4}$ The present authors were unable to draw any conclusion about adherence over the course of ET because of the short duration of follow-up in this study.

Several studies have evaluated the association between adherence and patient-related factors ${ }^{2,5}$ In a cross-sectional survey, the risk factors associated with non-adherence were comorbid conditions, polypharmacy and younger age. ${ }^{2}$ In the current cohort, age and whether the patient was taking other medications were not significantly associated with adherence. However, the present study has some limitations. The use of a selfadministered questionnaire, as there was no objective measure of adherence for more accurate assessment, might have resulted in an overestimation of adherence. In addition, there was a small number of patients that had taken ET for more than three years and the response rate was not calculated. Furthermore, the included patients were on ET for more than one month which might affect the adherence as the behaviours of patients would change over the period of treatment.

This study found a relatively high level of adherence to ET among patients with BC. However, there was a gap in the counselling provided by pharmacists. Further studies should be conducted to assess adherence for a longer duration and to further investigate the factors that may be associated with non-adherence such as socioeconomic status.

\section{AUTHORS' CONTRIBUTION}

NF and KA conceptualised and designed the manuscript. AG, MD, RA, HS, HS and RR collected and assembled the data. KA analysed the data. NF, AG and KA wrote the manuscript. All authors approved the final version of the manuscript.

\section{"Nour Faqeer, ${ }^{1}$ Alia Al Gharabli, ${ }^{1}$ Maha Dalbah, ${ }^{1}$ Khawalah Ammar, ${ }^{2}$ Randa Agha, ${ }^{1}$ Hala Shannies, ${ }^{1}$ Hala Sroji, ${ }^{1}$ Rawaa Al Rabie ${ }^{1}$}

${ }^{1}$ Department of Pharmacy, King Hussein Cancer Center, Amman, Jordan; ${ }^{2}$ Office of Scientific Affairs and Research, King Hussein Cancer Center, Amman, Jordan

*Corresponding Author's e-mail: nfaker@khcc.jo

\section{References}

1. Hershman DL, Shao T, Kushi LH, Buono D, Tsai WY, Fehrenbacher L, et al. Early discontinuation and non-adherence to adjuvant hormonal therapy are associated with increased mortality in women with breast cancer. Breast Cancer Res Treat 2011; 126:529-37. https://doi. org/10.1007/s10549-010-1132-4.

2. Pourcelot C, Orillard E, Nallet G, Dirand C, Billion-Rey F, Barbier G, et al. Adjuvant hormonal therapy for early breast cancer: An epidemiologic study of medication adherence. Breast Cancer Res Treat 2018; 169:153-62. https://doi.org/10.1007/s10549-018-4676-3.

3. Ali EE, Cheung KL, Lee CP, Leow JL, Yap KY, Chew L. Prevalence and determinants of adherence to oral adjuvant endocrine therapy among breast cancer patients in Singapore. Asia Pac J Oncol Nurs 2017; 4:283-9. https://doi.org/10.4103/2347-5625.212864. 
4. Robinson B, Dijkstra B, Davey V, Tomlinson S, Frampton C. Adherence to adjuvant endocrine therapy in Christchurch women with early breast cancer. Clin Oncol (R Coll Radiol) 2018; 30:e9-15. https://doi.org/10.1016/j.clon.2017.10.015.

5. Ziller V, Kalder M, Albert US, Holzhauer W, Ziller M, Wagner U, et al. Adherence to adjuvant endocrine therapy in postmenopausal women with breast cancer. Ann Oncol 2009; 20:431-6. https://doi.org/10.1093/annonc/mdn646. 